\title{
Abrupt formation of intracardiac thrombus during cardiopulmonary bypass with full heparinization -A case report-
}

\author{
Seong-Hyop Kim ${ }^{1}$, Jae-Sung Ryu ${ }^{1}$, Tae-Yop Kim ${ }^{1}$ Tae-Gyoon Yoon ${ }^{1}$, Woonseok Kang ${ }^{1}$, and \\ Ji Eun Song ${ }^{2}$
}

Department of Anesthesiology and Pain Medicine, ${ }^{1}$ Konkuk University Hospital, Seoul, ${ }^{2} \mathrm{CHA}$ Bundang Medical Center, CHA University, Seongnam, Korea

Intracardiac thrombus during cardiopulmonary bypass (CPB) with full heparinization is very rare but fatal. A 60-year-old woman was scheduled for aortic and mitral valve repairs with a maze procedure for mixed aortic and mitral valvular heart disease with atrial fibrillation. Preoperative transthoracic echocardiography and cardiac computed tomography showed moderate aortic regurgitation and moderate mitral stenosis with regurgitation. There was no intracardiac thrombus. Aortic and mitral valve repairs with the maze procedure were successfully performed without unexpected events. During CPB weaning, a mobile hyper-echogenic mass in the left atrium was detected on transesophageal echocardiography. After cardiac arrest, it was surgically removed. On completion of the operation, weaning from $\mathrm{CPB}$ was accomplished uneventfully. The patient fully recovered and was discharged from the intensive care unit on her third postoperative day. (Korean J Anesthesiol 2012; 62: 175-178)

Key Words: Cardiopulmonary bypass, Thrombosis, Transesophageal echocardiography.

Intracardiac thrombus during cardiopulmonary bypass (CPB) with full heparinization is rare but can be associated with serious complications because of its potential propagation in the direction of blood flow. The arterial embolism may lead to permanent damages, resulting in stroke, infarction of organs, or gangrene of extremities, commonly the lower extremities.

The diagnosis of intracardiac thrombus is difficult with clinical symptoms and signs while the patient is under general anesthesia. There is no definite way to diagnose intracardiac thrombus intraoperatively without transesophageal echocardiography (TEE).

Here we report a case of intracardiac thrombus that occurred during $\mathrm{CPB}$ with full heparinization in a patient undergoing aortic and mitral valve repairs with the maze procedure. We quickly diagnosed intracardiac thrombus with TEE and prevented the complications associated with it.

Received: March 21, 2011. Revised: May 2, 2011. Accepted: May 19, 2011.

Corresponding author: Ji Eun Song, M.D., Department of Anesthesiology and Pain Medicine, CHA Bundang Medical Center, CHA University, Yatap-dong, Bundang-gu, Seongnam 463-712, Korea. Tel: 82-31-780-5432, Fax: 82-31-780-5566, E-mail: queenlucy20@naver.com (c) This is an open-access article distributed under the terms of the Creative Commons Attribution Non-Commercial License (http:// creativecommons.org/licenses/by-nc/3.0/), which permits unrestricted non-commercial use, distribution, and reproduction in any medium, provided the original work is properly cited. 


\section{Case Report}

A 60-year-old woman (height, $150 \mathrm{~cm}$; weight, $58 \mathrm{~kg}$ ) was scheduled for aortic and mitral valve repairs with maze procedure for mixed aortic and mitral valvular heart disease with atrial fibrillation (AF). She had taken digoxin, furosemide, atenolol, and warfarin for 6 months. Warfarin was stopped for 5 days before surgery. The preoperative electrocardiogram showed AF with ventricular rates of $60-100 / \mathrm{min}$ and left ventricular hypertrophy. Chest X-ray showed cardiomegaly and pleural effusion. Transthoracic echocardiography (TTE) and cardiac computed tomography (CT) revealed an ejection fraction of normal range and a dilated left atrium (LA) and ventricle, as well as moderate aortic regurgitation and moderate mitral stenosis and regurgitation. There was no intracardiac thrombus. Before the operation, coagulation profiles were as follows: prothrombin time (PT) $14.9 \mathrm{~s}$, international normalized ratio (INR) 1.14, activated partial thromboplastin time (aPTT) $35.2 \mathrm{~s}$, and fibrinogen $255 \mathrm{mg} / \mathrm{dl}$.

Upon arrival at the operation room, after establishing routine invasive arterial blood pressure and noninvasive patient monitoring (electrocardiography, pulse oximetry, bispectral index, and cerebral oximetry), anesthesia was induced and maintained using target-controlled infusion of propofol (target concentration, $1.2-1.3 \mu \mathrm{g} / \mathrm{ml}$ ) and remifentanil (target concentration, $10-20 \mathrm{ng} / \mathrm{ml}$ ). Muscle relaxation was achieved with the administration of a bolus of rocuronium under the guidance of peripheral monitoring of neuromuscular transmission. Then a pulmonary artery catheter (Swan-Ganz CCOmbo CCO $/ \mathrm{SvO}_{2}{ }^{\circledR}$, Edwards Lifesciences, Irvine, CA, USA) was inserted after anesthesia induction. TEE probe (6T probe $^{\mathrm{TM}}$, GE Healthcare, Waukesha, WI, USA) was inserted. TEE (Vivid $7^{\mathrm{TM}}$, GE Healthcare, Waukesha, WI, USA) revealed no

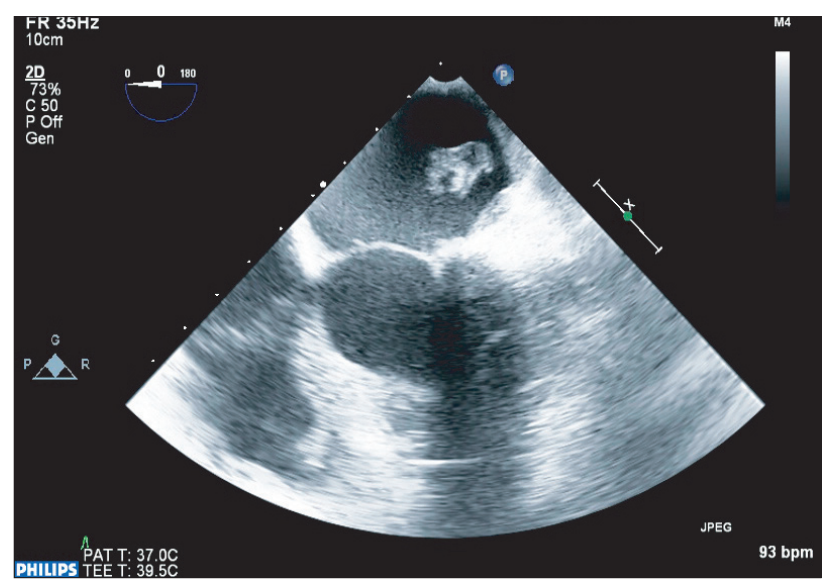

Fig. 1. The mid-esophageal four-chamber view. The hyper-echogenic mass in the left atrium is shown. intracardiac thrombus, as was seen in the preoperative TTE and cardiac CT.

The activated clotting time (ACT) before CPB was 140 s. CPB was initiated after heparin 300 units $/ \mathrm{kg}$ was injected, and ACT above $450 \mathrm{~s}$ was achieved. During CPB for $211 \mathrm{~min}$, the ACT was checked every $60 \mathrm{~min}$ and maintained above $450 \mathrm{~s}$ with additional heparin 2,000 units given 60 min after CPB started. Aortic and mitral valve repairs using bovine pericardium, a mitracon strip (Mitr-lift, Sciencity, Wonju-Shi, Kangwon-do, Korea), and a sinotubular junction ring, and following the maze procedure, were successfully performed without unexpected events. Defibrillation with $50 \mathrm{~J}$ was applied and TEE was performed to evaluate aortic and mitral valve and regional wall motion abnormality. There were no specific findings, including no thrombosis. The core temperature reached $36^{\circ} \mathrm{C}$ and weaning of CPB was attempted. At that time, a mobile hyper-echogenic mass in the LA was detected via TEE (Fig. 1). The mass seemed to be a thrombus. It had a distinct border and different echogenic density than that of blood or the atrial wall. Its movement was separate from that of the atrial wall. At that point, the ACT was checked and recorded at $504 \mathrm{~s}$. Rotation thromboelastometry (ROTEM ${ }^{\circledR}$, TEM Innovations, Munich, Bayern, Germany) showed hypocoagulability, with clotting time (CT) of $972 \mathrm{sec}$ (normal 100-240 sec), clot formation time (CFT) of $330 \mathrm{sec}$ (normal 30-110 sec), $\alpha$-angle $33.5^{\circ}$ (normal 70-83 ${ }^{\circ}$ ), and maximum clot firmness (MCF) of $49.8 \mathrm{~mm}$ (normal 50$72 \mathrm{~mm}$ ). After discussions with the cardiac surgeons, CPB was re-started for surgical confirmation and proper management of a mobile hyper-echogenic mass. Left atriotomy revealed that the mass was a thrombus, $15 \times 20 \mathrm{~mm}$ in size with an irregular spherical surface (Fig. 2).

The second CPB weaning was successful. Protamine was injected for neutralization of heparin and an ACT of $124 \mathrm{~s}$ was

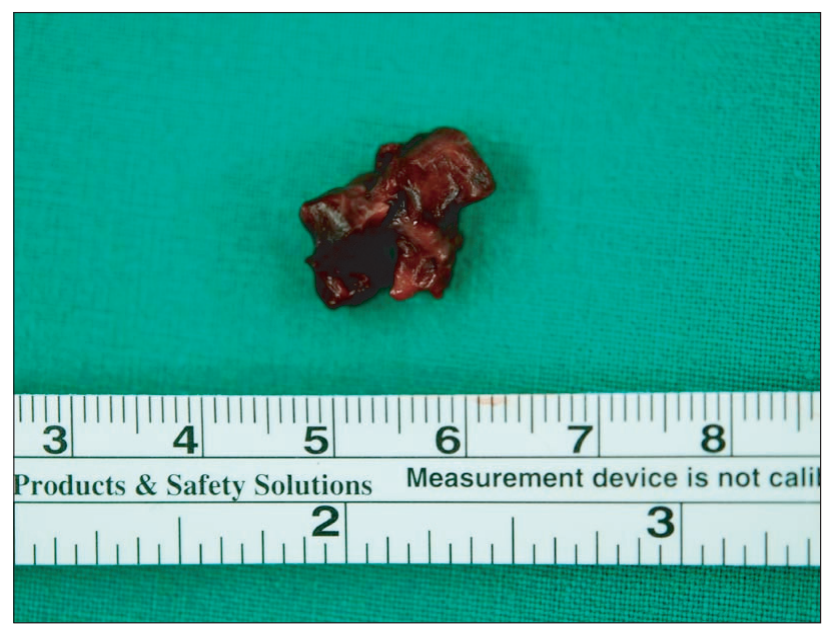

Fig. 2. The surgically removed intracardiac thrombus. 
recorded. Total CPB time was $246 \mathrm{~min}$. During $\mathrm{CPB}$, three units of packed red blood cells (RBCs) were transfused. After weaning from $\mathrm{CPB}$, eight units of platelet concentrates, three units of fresh frozen plasma (FFP), and three units of RBCs were transfused. On completion of the operation, weaning from CPB was accomplished uneventfully. The patient was transferred to the intensive care unit (ICU). In the ICU, mild coagulopathy was observed with PT $18.1 \mathrm{~s}$, INR 1.46, aPTT $35.2 \mathrm{~s}$, fibrinogen $189 \mathrm{mg} / \mathrm{dl}$, D-dimer $5.40 \mathrm{mcg} / \mathrm{ml}$, and platelets $72000 / \mathrm{mcl}$. After 3 days, the patient made an uneventful recovery and was transferred to a general ward.

\section{Discussion}

Intracardiac thrombus during or just after CPB have rarely been reported. In one such rare case, Gillham and Tousignant [1] reported severe acute thrombosis of a mechanical aortic valve prosthesis while the patient was fully heparinized on CPB. Multiple interacting prothrombotic factors, such as use of aprotinin [2-4], acquired antithrombin III deficiency, prolonged duration of CPB [5], post-protamine status [4], transfusion of blood components, disseminated intravascular coagulation [6], preoperative congestive heart failure [2], biological glue [1], and multiple prosthetic surfaces [5], may contribute to intracardiac thrombus during or just after CPB. The report by Gillham and Tousignant [1] was associated with the use of biological glue. In addition, the patient was resistant to heparin and an antifibrinolytic drug was also administered.

In our case, aprotinin was not administered, nor were any antifibrinolytic drugs, and the patient had no known coagulation disorders. Contrary to our expectations, the rotation thromboelastometry showed hypocoagulability. No blood components, except RBCs, were transfused before CPB weaning. Biological glue for aortic and mitral valve repairs was not used. Therefore, the prolonged duration of CPB (211 min) might have contributed to the intracardiac thrombus in this case. Congested pulmonary venous blood, related to mitral regurgitation, might become sticky during an extended $\mathrm{CPB}$. The patient had no right-to-left cardiac shunt, such as a patent foramen ovale. Preoperative examinations did not show intracardiac thrombus. Therefore, the intracardiac thrombus would have originated from the left cardiac chamber itself or the pulmonary veins.

Sudden development of refractory hypotension with a rapid rise in pulmonary artery pressures within $10 \mathrm{~min}$ after infusion of protamine and while closing the sternum might be key signs of right heart thrombus [6]. However, left heart thrombus cannot be detected until serious complication occurs.

Transesophageal echocardiography is a useful tool for the diagnosis and evaluation of intracardiac thrombus. In particular, the evaluation of posterior structures of the heart, such as the LA and left atrial appendage (LAA), is much easier with TEE. The sensitivity and specificity of TEE for thrombus in the LA have been reported as $93-100 \%$ and $99-100 \%$ [7-9], respectively.

However, it is important to distinguish between intracardiac thrombus and artifacts or pitfalls during TEE examination. Kim et al. [10] reported that folding of the right atrium appeared as a mass-like thrombus via TEE. Pressman and Figueredo [11] also reported misdiagnosis of the inverted LAA as an LA thrombus. A large hypertrophic trabecula [12], stagnant blood, or a warfarin ridge can also be confused for an intracardiac thrombus. In our case, however, the mobile hyper-echogenic mass with a clearcut margin was determined conclusively to be a thrombus. The mass was separate from the adjacent LA wall, so it could be distinguished from pectinate muscle, trabeculations, and inverted LAA. It could be differentiated from suture materials because it had no connection with the ring of the mitral valve annuloplasty and was less echogenic than suture materials.

In conclusion, abrupt formation of intracardiac thrombus with full heparinization can occur. For early detection of an intracardiac thrombus and prevention of detrimental outcomes, meticulous attention and close observation with TEE is required.

\section{References}

1. Gillham MJ, Tousignant CP. Diagnosis by intraoperative transesophageal echocardiography of acute thrombosis of mechanical aortic valve prosthesis associated with the use of biological glue. Anesth Analg 2001; 92: 1123-5.

2. Cooper JR Jr, Abrams J, Frazier OH, Radovancevic R, Radovancevic B, Bracey AW, et al. Fatal pulmonary microthrombi during surgical therapy for end-stage heart failure: possible association with antifibrinolytic therapy. J Thorac Cardiovasc Surg 2006; 131: 963-8.

3. Schmitz A, Hartmann M. Acute intracardiac thrombus formation during thoracoabdominal aortic surgery. Anesth Analg 2006; 102: 1658-9.

4. Heindel SW, Mill MR, Freid EB, Valley RD, White GC 2nd, Norfleet EA. Fatal thrombosis associated with a hemi Fontan procedure, heparin protamine reversal, and aprotinin. Anesthesiology 2001; 94: 369-71.

5. Neira VM, Sawchuk C, Bonneville KS, Chu V, Warkentin TE. Case report: management of immediate post-cardiopulmonary bypass massive intra-cardiac thrombosis. Can J Anaesth 2007; 54: 461-6.

6. Augoustides JG, Kilbaugh T, Harris H, Glick JH, Acker M, Savino JS. Fatal thrombosis after mitral valve replacement for endocarditis: aprotinin and disseminated intravascular coagulation (Letter). Anesthesiology 2006; 104: 213.

7. Manning WJ, Weintraub RM, Waksmonski CA, Haering JM, Rooney PS, Maslow AD, et al. Accuracy of transesophageal echocardiography for identifying left atrial thrombi. A prospective, intraoperative study. Ann Intern Med 1995; 123: 817-22. 
8. Hwang JJ, Chen JJ, Lin SC, Tseng YZ, Kuan P, Lien WP, et al. Diagnostic accuracy of transesophageal echocardiography for detecting left atrial thrombi in patients with rheumatic heart disease having undergone mitral valve operations. Am J Cardiol 1993; 72: 677-81.

9. Suriani RJ, Neustein S, Shore-Lesserson L, Konstadt S. Intraoperative transesophageal echocardiography during noncardiac surgery. J Cardiothorac Vasc Anesth 1998; 12: 274-80.
10. Kim SH, Yoon TG, Kim TY. Folding of right atrium misdiagnosed as a thrombus after mitral valve replacement - A case report -. Korean J Anesthesiol 2008; 54: 566-8.

11. Pressman GS, Figueredo VM. Development of an intraoperative left atrial thrombus? J Am Coll Cardiol 2009; 54: el.

12. Delange Segura L. Limitations of transesophageal ultrasound in the assessment of intracardiac masses: a case report. Rev Esp Anestesiol Reanim 2005; 52: 421-4. 